\title{
Analytic solution for fluxons in a long Josephson junction with surface losses
}

\section{Sakai, S.; Pedersen, Niels Falsig}

\section{Published in:}

Physical Review B

Link to article, DOI:

10.1103/PhysRevB.34.3506

Publication date:

1986

Document Version

Publisher's PDF, also known as Version of record

Link back to DTU Orbit

Citation (APA):

Sakai, S., \& Pedersen, N. F. (1986). Analytic solution for fluxons in a long Josephson junction with surface losses. Physical Review B, 34(5), 3506-3509. https://doi.org/10.1103/PhysRevB.34.3506

\section{General rights}

Copyright and moral rights for the publications made accessible in the public portal are retained by the authors and/or other copyright owners and it is a condition of accessing publications that users recognise and abide by the legal requirements associated with these rights.

- Users may download and print one copy of any publication from the public portal for the purpose of private study or research.

- You may not further distribute the material or use it for any profit-making activity or commercial gain

- You may freely distribute the URL identifying the publication in the public portal

If you believe that this document breaches copyright please contact us providing details, and we will remove access to the work immediately and investigate your claim. 


\title{
Analytic solution for fluxons in a long Josephson junction with surface losses
}

\author{
S. Sakai* and N. F. Pedersen \\ Physics Laboratory I, The Technical University of Denmark, DK-2800 Lyngby, Denmark
}

(Received 24 March 1986)

\begin{abstract}
Analytic solutions for a fluxon in a long Josephson junction in the presence of surface losses ( $\beta$ term) as well as shunt losses ( $\alpha$ term) are obtained by assuming a triangular current-phase relation. This theoretical result provides exact information on fluxon properties (e.g., the line shape, velocity, etc.), independent of the magnitude of $\alpha$ and $\beta$. We find that if $\beta$ is smaller than a critical value, the fluxon behavior is similar to that of the $\beta=0$ case, but if $\beta$ is larger, quite different behavior is observed, particularly in the high-velocity region.
\end{abstract}

There has been considerable interest in the effect of surface losses on fluxons (i.e., solitons in long Josephson junctions). A recent numerical work ${ }^{1}$ on long annular Josephson junctions demonstrated that new features, not expected from the perturbation theory, ${ }^{2}$ are created by the surface loss: (i) Lorentz contraction is greatly reduced or even eliminated, (ii) oscillations may appear on the trailing edge of the fluxon, and (iii) for high bias the fluxon velocity is not asymptotic to the velocity of light.

In this paper we present an analytical method for $2 \pi-$ soliton solutions of the traveling-wave type, and derive many significant effects of the surface losses. The theory is based on assuming a triangular current-phase relation and extending a previously published model calculation ${ }^{3}$ to a third-order system. The advantage of the theory is that it does not require small perturbing terms. In physical systems such large damping has been realized by having resistors together with the long Josephson junction. ${ }^{4}$

Fluxon motion in the long Josephson junction with uniform bias current $\eta$ is assumed to be described by

$$
-\phi_{x x}+\phi_{t t}+f(\phi)=\eta-\alpha \phi_{t}+\beta \phi_{x x t} \text {. }
$$

Here $f(\phi)$ is the current-phase relation and normally $f(\phi)=\sin \phi$ is assumed. The term $\beta \phi_{x x t}$ describes the surface losses, and $\alpha \phi_{t}$ describes the shunt losses. The normalizations and a further description of Eq. (1) may be found in Ref. 2.

Here we are interested in traveling-wave solutions of the form $\phi=\phi(\xi)$ where $\xi=x-u t$. Equation (1) may be transformed into the ordinary differential equation (ODE),

$$
-\beta u \phi_{\xi \xi \xi}+\left(1-u^{2}\right) \phi_{\xi \xi}+\alpha u \phi_{\xi}-f(\phi)+\eta=0 .
$$

With $f(\phi)=\sin \phi$, Eq. (2) cannot be solved analytically, not even with $\beta=0$. To solve Eq. (2) analytically we will assume a triangular current-phase relation, i.e.,

$f(\phi)=\left\{\begin{array}{l}k(\phi-2 n \pi),-\pi / 2+2 n \pi \leqq \phi<\pi / 2+2 n \pi, \\ -k(\phi-\pi-2 n \pi), \pi / 2+2 n \pi \leqq \phi<3 \pi / 2+2 n \pi .\end{array}\right.$

In the later numerical examples, we will set $k=2 / \pi$ to have unit amplitudes of $f(\phi)$. The current-phase relation, Eq. (3), is shown in Fig. 1 together with a schematic draw- ing of a topological $2 \pi$ soliton. As seen from the figure, region I corresponds to the leading edge of the fluxon, region III to the trailing edge, and region II to the core. Because of translational symmetry the joining of regions II and III may be assumed to happen at $\xi=0$. The joining between regions I and II happens at $\xi=\xi_{1}$ as indicated in the figure.

The method of solution is the following. In each of the three regions in Eqs. (2) and (3) is a linear ODE of third order. The solutions are found by standard methods and the solutions for the different regions $\left(\phi_{1}, \phi_{2}\right.$, and $\left.\phi_{3}\right)$ are joined at $\xi=0$ and $\xi=\xi_{1}$ by requiring $\phi, \phi_{\xi}$, and $\phi_{\xi \xi}$ to be continuous. Further, boundary conditions at $\xi \rightarrow \pm \infty$ are imposed to give a unique solution $\phi(\xi)$.

The linear ODE's [Eqs. (2) and (3)] are solved by finding the roots of the characteristic polynomial $P_{+}$in regions $I$ and III, and $P-$ in region II where $P_{ \pm}$are given by

$$
P_{ \pm}(x)=\beta u x^{3}-\left(1-u^{2}\right) x^{2}-\alpha u x \pm k=0 .
$$

These roots are obtained by standard methods. ${ }^{5}$ In order to provide qualitative insight, it is convenient to define the functions $h_{+}$(regions I and III) and $h_{-}$(region II).

$$
h_{ \pm}(u)=\frac{2 p^{3} \pm\left(1-u^{2}\right)\left(2 p^{2}+3 \alpha \beta u^{2}\right)}{27 \beta^{2} u^{2}}
$$

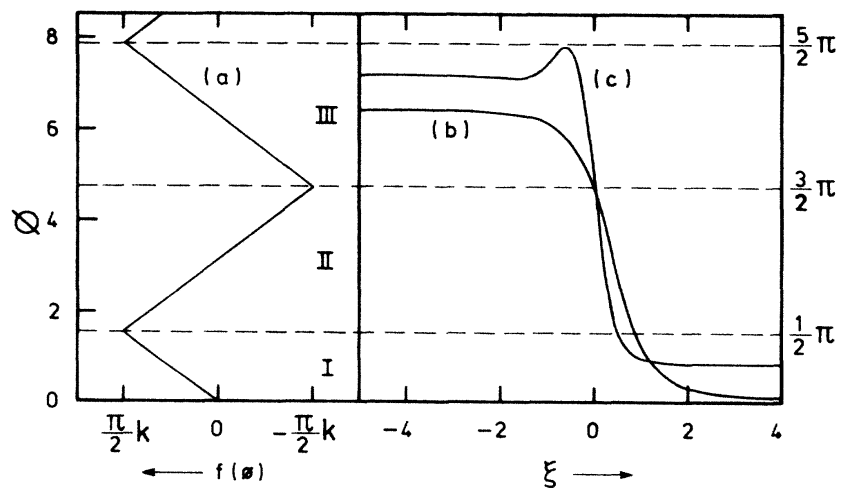

FIG. 1. (a) The triangular current-phase relation $f(\phi)$. (b) and (c) Fluxon line shapes with $\alpha=0.02, \beta=0.01$, and $k=2 / \pi$. (b) $u=0.9$ giving $\eta=0.079$. (c) $u=1.0$ giving $\eta=0.569$. 
TABLE I. Roots of the characteristic polynomial, $P_{+}=0$ for regions I and III.

\begin{tabular}{llcc}
\hline \hline$k<h_{+}$ & $q_{1}<0$ & $q_{2}>0$ & $q_{3}>0$ \\
$k>h_{+}$ & $q_{1}<0$ & $q_{2}=q_{a}+i q_{b}$ & $q_{3}=q_{a}-i q_{b}$ \\
\hline
\end{tabular}

with $p=\left[\left(1-u^{2}\right)^{2}+3 \alpha \beta u^{2}\right]^{1 / 2}$. The roots of $P_{+}(x)=0$ are called $q_{1}, q_{2}$, and $q_{3}$. Their properties may be seen in Table I $\left(q_{a}>0\right)$.

Using the boundary condition $\phi_{1} \rightarrow \eta / k$ for $\xi \rightarrow \infty$ in region I and $\phi_{3} \rightarrow \eta / k+2 \pi$ for $\xi \rightarrow-\infty$ in region III, we find

$\phi_{1}=A_{1} \exp \left(q_{1} \xi\right)+\frac{\eta}{k}$, region I ,

$\phi_{3}=C_{2} \exp \left(q_{2} \xi\right)+C_{3} \exp \left(q_{3} \xi\right)+\frac{\eta}{k}+2 \pi$, region III ,

where $A_{1}, C_{2}$, and $C_{3}$ are arbitrary constants.

We note from Eq. (6) and Table $I$ that $\phi_{1}$ is always exponentially damped in region $\mathrm{I}$; however, if $h_{+}(u)<k$, it may contain a damped oscillation at the trailing edge (region III), because $\phi_{3}$ may be expressed as

$$
\begin{aligned}
\phi_{3}=2 \exp \left(q_{a} \xi\right) & {\left[\operatorname{Re}\left(C_{2}\right) \cos \left(q_{b} \xi\right)\right.} \\
& \left.-\operatorname{Im}\left(C_{2}\right) \sin \left(q_{b} \xi\right)\right]+\eta / k+2 \pi .
\end{aligned}
$$

For region II the behavior of $\phi$ is determined by the roots, $r_{1}, r_{2}$, and $r_{3}$ of $P-(x)=0$ [Eq. (4)]. Those roots are characterized in Table II $\left(r_{a}<0\right)$. The solution in region II may be written by

$\phi_{2}=B_{1} \exp \left(r_{1} \xi\right)+B_{2} \exp \left(r_{2} \xi\right)+B_{3} \exp \left(r_{3} \xi\right)-\eta / k+\pi$.

The constants $B_{1}, B_{2}$, and $B_{3}$ are determined together with $A_{1}, C_{2}, C_{3}, \eta$, and $\xi_{1}$ by joining the solutions and their first and second derivatives at $\phi(0)=3 \pi / 2$ and $\phi\left(\xi_{1}\right)=\pi / 2$. After lengthy calculations, we obtain fairly simple results. We define the following quantities based on the roots of Eq. (4),

$$
\begin{aligned}
a_{i} & =q_{2} q_{3}+r_{i}\left(q_{2}+q_{3}\right)-r_{i}^{2}, i=1,2,3, \\
b_{i} & =\frac{r_{j} r_{k}+q_{1}\left(r_{j}+r_{k}\right)-q_{1}^{2}}{\left(r_{j}-r_{i}\right)\left(r_{i}-r_{k}\right)},\left(\begin{array}{l}
i \\
j \\
k
\end{array}\right)=\left(\begin{array}{l}
1 \\
2 \\
3
\end{array}\right),\left(\begin{array}{l}
2 \\
3 \\
1
\end{array}\right),\left(\begin{array}{l}
3 \\
1 \\
2
\end{array}\right) .
\end{aligned}
$$

The procedure is now the following. With $\alpha$ and $\beta$ as parameters and $u$ as the independent variable, the roots $q_{1}$, $q_{2}, q_{3}$, and $r_{1}, r_{2}, r_{3}$ to Eq. (4) are determined. $\xi_{1}$ is determined as a positive root $^{6}$ to

$$
\sum_{i=1}^{3} a_{i} b_{i} \exp \left(-r_{i} \xi_{1}\right)=0
$$

and $\eta(u)$ is determined from

$$
\eta=\frac{\pi k}{2} \frac{\sum_{i=1}^{3} b_{i} \exp \left(-r_{i} \xi_{1}\right)-1}{\sum_{i=1}^{3} b_{i} \exp \left(-r_{i} \xi_{1}\right)+1}
$$

TABLE II. Roots of the characteristic polynomial, $\boldsymbol{P}_{-}=0$ for region II.

\begin{tabular}{lccc}
\hline \hline$k<h-$ & $r_{1}<0$ & $r_{2}<0$ & $r_{3}>0$ \\
$k>h-$ & $r_{1}=r_{a}-i r_{b}$ & $r_{2}=r_{a}+i r_{b}$ & $r_{3}>0$ \\
\hline \hline
\end{tabular}

Finally, the constants $A_{1}, B_{1}, B_{2}, B_{3}, C_{2}$, and $C_{3}$ are calculated by

$$
\begin{aligned}
& A_{1}=\left(\frac{\pi}{2}-\frac{\eta}{k}\right) \exp \left(-q_{1} \xi_{1}\right), \\
& B_{i}=\left(\frac{\pi}{2}-\frac{\eta}{k}\right) b_{i} \exp \left(-r_{i} \xi_{1}\right), i=1,2,3, \\
& C_{i}=\sum_{k=1}^{3} c_{i k} B_{k}, i=2,3,
\end{aligned}
$$

with

$$
c_{i k}=\frac{r_{k}\left(q_{j}-r_{k}\right)}{q_{i}\left(q_{j}-q_{i}\right)},\left(\begin{array}{l}
i \\
j
\end{array}\right)=\left(\begin{array}{l}
2 \\
3
\end{array}\right),\left(\begin{array}{l}
3 \\
2
\end{array}\right) .
$$

Figure 2 shows some examples of calculated fluxon line shapes with $k=2 / \pi$. Note that the logarithm of the absolute value of $\phi_{\xi}$ is shown. Both the front and the tail of the fluxon show an exponential behavior. For very small $\beta$ $\left(=10^{-6}\right)$ a substantial Lorentz contraction is observed [Fig. 2(a)]. With a $\beta$ term present no Lorentz contraction occurs [Figs. 2(b) and 2(c)]. For large velocity (high $\eta$ ) and $\beta$ losses present [Fig. 2(c)], the spatially damped oscillation on the trailing edge [Eq. (7)] is clearly seen. The corresponding behavior of the phase is shown in Fig. 1(c). These qualitative features were also found in the numerical simulations with a sinusoidal current-phase relation in Ref. 1.

From Eqs. (10) and (11) the $\eta$ vs $u$ relations are obtained. Figure 3 shows the $\eta$ vs $u$ curves for $\alpha=0.1$ and

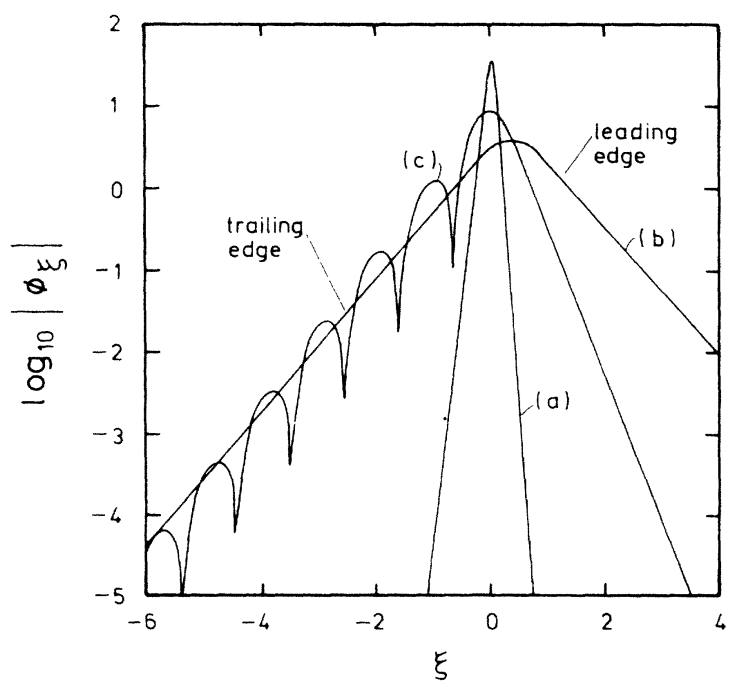

FIG. 2. Fluxon line shapes with $k=2 / \pi$ and $\alpha=0.02$. (a) $\beta=0.000001, u=0.999$ giving $\eta=0.478$; (b) $\beta=0.01, u=0.9$ giving $\eta=0.079$; and (c) $\beta=0.01, u=1.0$ giving $\eta=0.569$. 


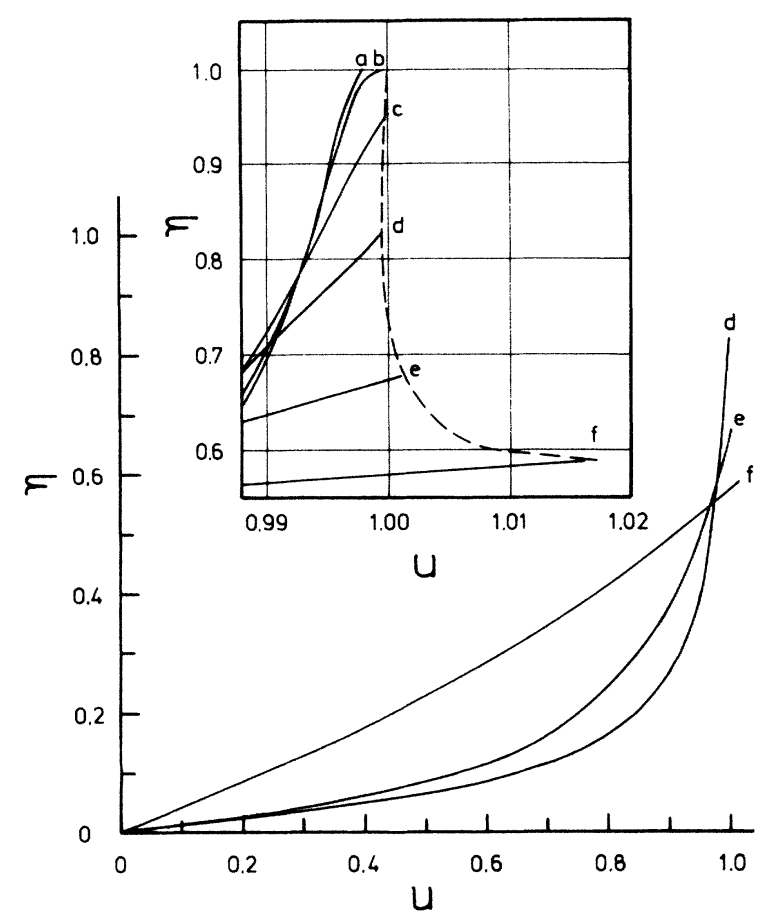

FIG. 3. Velocity vs bias curves with $k=2 / \pi$ and $\alpha=0.1$. $\beta=$ (a) $10^{-6}$, (b) 0.00028 , (c) 0.002 , (d) 0.01 , (e) 0.1 , and (f) 1.0 .

various values of $\beta$. The inset of the figure shows the enlargement around $u=1$.

The functions $h \pm$ [Eq. (5)] are useful to estimate the qualitative behavior of fluxons. Figure 4 shows examples for two sets of damping parameters. At $u=1$ we have

$$
h_{+}=h_{-}=\frac{2}{3 \sqrt{3}}\left(\frac{\alpha^{3}}{\beta}\right)^{1 / 2} \equiv h_{0} \text {. }
$$

Depending on the magnitude of $h_{0} / k$, two cases occur.

(a) For $\beta \leqq 4 \alpha^{3} / 27 k^{2}\left(k \leqq h_{0}\right)$ we find a similar qualitative behavior as for the $\beta=0$ case $^{3,7}$ such as the following: (1) no oscillations appear, because $q_{1}, q_{2}$, and $q_{3}$ are always real numbers; (2) at $u=u_{c 1}(<1), \eta_{\max }$ goes to 1 , where $\eta_{\max }$ is the maximum of $\eta$, and $u_{c 1}$ is the root of $h_{-}(u)=k$. This second feature may be understood from the following: If $h_{-}(u) \rightarrow k$, then $r_{1} \rightarrow r_{2}$ and $\left|b_{1}\right|$ and $\left|b_{2}\right| \rightarrow \infty$ from Eq. (9), which lead to $\eta \rightarrow 1$ from Eq. (11). Here $k=2 / \pi$ is used again. For $u_{c 1}<u \leqq 1$, we have no solutions because actual calculations show that there are no roots of Eq. (10). The inset of Fig. 3 shows that, as $\beta$ increases from 0 to $4 \alpha^{3} / 27 k^{2}, u_{c 1}$ moves $^{3}$ from $1 /\left(1+\alpha^{2} / 4 k^{2}\right)^{1 / 2}$ to 1 .

(b) For $\beta>4 \alpha^{3} / 27 k^{2}\left(k>h_{0}\right)$ we find changes in the qualitative behavior such as the following: (1) for $u_{c 2}<u$,

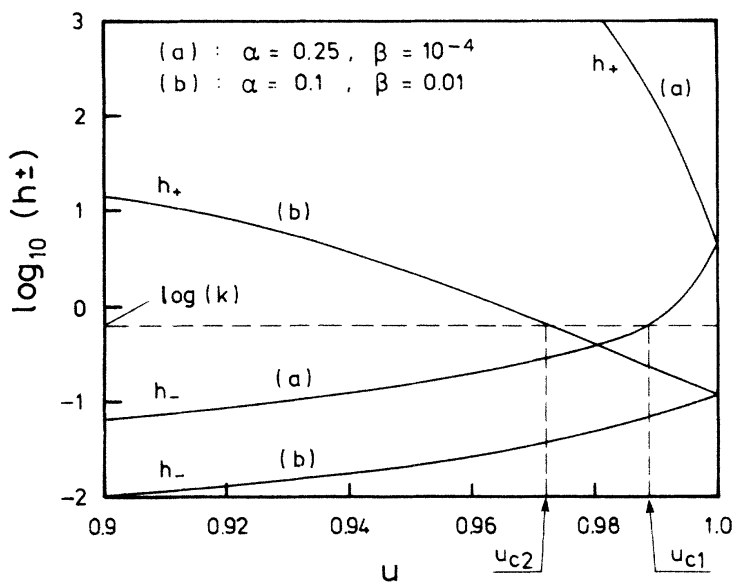

FIG. 4. $h_{ \pm}$vs $u$ relation, where $u_{c 1}$ is the root of $h-(u)=k$ and $u_{c 2}$ is the root of $h_{+}(u)=k$. If $\beta \leqq 4 \alpha^{3} / 27 k^{2}$, at $u=u_{c 1}, \eta$ reaches $\eta_{\max }=1$. If $\beta>4 \alpha^{3} / 27 k^{2}$, at $u>u_{c 2}$ oscillations appear on the trailing edge of fluxon line shapes.

oscillations appear on the trailing edge as shown in Figs. 1 and 2, where $u_{c 2}$ is the root of $h_{+}(u)=k$; (2) when $u$ approaches $1, \eta$ does not reach 1 . The numerical work ${ }^{1}$ assuming the sinusoidal current-phase relation in which $\alpha$ and $\beta$ always satisfied $\beta>4 \alpha^{3} / 27 k^{2}$, showed the same features.

Under the oscillation conditions, our analytical method can be applied up to the states where the maximum peak of the oscillation just reaches $5 \pi / 2$. The dashed curve in the inset of Fig. 3 represents this limit. Note that the curve $u=1$ is not related to this limit. For small $\beta$ (but $\beta>4 \alpha^{3} / 27 k^{2}$ ), this limit velocity is very close to 1 but less than 1 . As $\beta$ increases, it exceeds $1 .^{8}$ The stability problem for this region will be treated in a future publication. ${ }^{9}$ We note here, however, that we found the solution at $\alpha=0.1, \beta=1.0$, and $u=1.015$ in Fig. 3 stable by direct computer simulation.

We have investigated fluxon propagation in a long Josephson junction in the presence of surface losses. By assuming a triangular current-phase relation it was possible to obtain an analtyical solution. The behavior of the solutions to this model is classified into the following two: If $\beta$ is smaller than a constant times $\alpha^{3}$, the qualitative behavior is similar to the $\beta=0$ case, but if $\beta$ is larger, quite different features from the $\beta=0$ case (e.g., damped oscillations of the trailing edge of a fluxon) appear.

One of us (S.S.) is grateful for the hospitality of the personnel in Physics Laboratory I, Technical University of Denmark, and for the support of the Science and Technology Agency of Japan.
*Permanent address: Electrotechnical Laboratory, 1-1-4 Umezono, Sakura-mura, Niihari-gun, Ibaraki 305, Japan.

${ }^{1}$ A. Davidson, N. F. Pedersen, and S. Pagano, Appl. Phys. Lett. 48, 1306 (1986).
${ }^{2}$ D. W. McLaughlin and A. C. Scott, Phys. Rev. A 18, 1652 (1978); A. Davidson, B. Dueholm, B. Kryger, and N. F. Pedersen, Phys. Rev. Lett. 55, 2059 (1985).

${ }^{3}$ S. Sakai and H. Tateno, Jpn. J. Appl. Phys. 22, 1374 (1983). 
${ }^{4}$ J. Nitta, A. Matsuda, and T. Kawakami, J. Appl. Phys. 55, 2758 (1984); H. Akoh, S. Sakai, A. Yagi, and H. Hayakawa, IEEE Trans. Magn. MAG-21, 737 (1985).

${ }^{5}$ See, for example, Handbook of Mathematical Functions, edited by M. Abramowitz and I. A. Stegun (Dover, New York, 1970).

${ }^{6}$ If $k>h_{-}$, there are more than one root, but only the smallest positive root is adopted. Other roots do not make solutions, because oscillating peaks of $\phi_{2}$ exceed $\pi / 2 \leqq \phi \leqq 3 \pi / 2$ (the range of region II). If $k<h_{-}$, the details are discussed in the text relating to Fig. 4.

${ }^{7}$ S. E. Burkov and A. E. Lifsic, Wave Motion 5, 197 (1983).

${ }^{8}$ Solutions with $u>1$ were pointed out in the numerical work of Nakajima et al., J. Appl. Phys. 45, 4095 (1974), although enough details to discuss the stability of the solutions were not given.

${ }^{9}$ S. Sakai and N. F. Pedersen (unpublished). 